\title{
The "multifaceted" onset of vasculitis neuropathy
}

\author{
Lorenzo Emmi • Danilo Squatrito · Giacomo Emmi • \\ Enrico Beccastrini $\cdot$ Elena Silvestri
}

Received: 21 November 2012/ Accepted: 3 December 2012/Published online: 20 December 2012

(C) SIMI 2012

The article from Lozeron et al. [1], published in this issue of Internal and Emergency Medicine, underscores the importance of recognizing or suspecting a systemic vasculitis in patients who complain of atypical or refractory sciatica-like symptoms.

Vasculitides is a group of systemic diseases characterized by a protean clinical presentation, usually with a pattern of multi-organ involvement, which if not promptly diagnosed, may be responsible for severe morbidity and mortality [2]. The following article reminds us that besides usual peripheral neurological presentations classically associated with systemic vasculitides, i.e., multifocal motor neuropathy or less frequently symmetric peripheral neuropathies, unusual neurological symptoms can in rare instances mark the disease onset [3].

Even if lumbosacral radiculoplexus neuropathy is mainly associated with microischemic events and diabetes mellitus, which was correctly ruled out in 8 of the study patients, it is also described in non-diabetic patients who probably suffered an underlying autoimmune microvasculitis process [4]. The possibility of an autoimmune lombosacral plexus radiculitis should be particularly looked for when sciatica-like symptoms, in non-diabetic patients, are not fully explained by radiological spine findings; or when the controlateral limb is subsequently involved, in a pattern ultimately resembling a multifocal neuropathy. Furthermore, these patients often undergo unnecessary and repeated spine surgery procedures.

L. Emmi $(\bowtie)$ - D. Squatrito · G. Emmi · E. Beccastrini ·

E. Silvestri

Center for autoimmune diseases, Lupus Clinic Unit,

Careggi Hospital, Florence, Italy

e-mail: 1.emmi@dmi.unifi.it
Besides the importance of a thorough history taking and physical examination, the authors underscore the role of correctly performing a nerve conduction study. This examination shows axonal damage and asymmetric multifocal involvement, giving an early basis for suspecting a vasculitis, and for performing subsequent investigations. The other fundamental step is a peripheral nerve biopsy, a very useful procedure especially when a vasculitis is suspected in patients with an atypical neurologic pattern of presentation. In this following study, biopsy specimens show fibrinoid necrosis in all patients, histologically supporting the classification criteria for diagnosing polyarteritis nodosa, confirming the well-known concept that this entity is probably the vasculitis most often associated with peripheral multifocal neuronal involvement $[5,6]$.

In summary, this article should remind us of what suspecting a rare disease in clinical practice means for doctors, and the importance of looking "beyond" common symptoms when these are atypical or refractory to standard treatment [7]. Regarding this last matter in particular, it is really worthwhile to underscore the fundamental role of tertiary referring centers for rare diseases, such as the center for rare peripheral neuropathies that conducted the study, since they probably represent the place where difficult-to-treat or refractory patients can have the best or the only chance to achieve a definitive and correct diagnosis.

Conflict of interest None.

\section{References}

1. Lozeron P, Lacroix C, Michon M, Theaudin M, Petit Lacour MC, Denier C, Adams D (2012) Vasculitis neuropathy mimicking lower limb mono-radiculopathy: a study and follow-up of 8 cases. Intern Emerg Med. doi:10.1007/s11739-012-0894-6 
2. Langford CA (2010) Vasculitis. J Allergy Clin Immunol $125(2$ Suppl 2):S216-S225

3. Dyck PJ, Norell JE, Dyck PJ (2001) Non-diabetic lumbosacral radiculoplexus neuropathy: natural history, outcome and comparison with the diabetic variety. Brain 124(Pt 6):1197-1207

4. Gorson KC (2007) Vasculitic neuropathies: an update. Neurologist 13(1):12-19
5. Said G (1997) Necrotizing peripheral nerve vasculitis. Neurol Clin 15(4):835-848

6. Columpsi D, Badini M, Scannella E, Montano N, Antivalle M, Tonolini M, Osio M (2010) A foot-drop case. Intern Emerg Med 5(4):321-324

7. Taruscio D, Capozzoli F, Frank C (2011) Rare diseases and orphan drugs. Ann Ist Super Sanita 47(1):83-93 